\title{
High-energy gamma-ray afterglows from low-luminosity gamma-ray bursts
}

\author{
Hao-Ning $\mathrm{He}^{1}$, Xiang-Yu Wang ${ }^{1,5}$, Yun-Wei Yu ${ }^{1,2}$ and Peter Mészáros ${ }^{3,4}$
}

\begin{abstract}
The observations of gamma-ray bursts (GRBs) such as 980425, 031203 and 060218, with luminosities much lower than those of other classic bursts, lead to the definition of a new class of GRBs - low-luminosity GRBs. The nature of the outflow responsible for them is not clear yet. Two scenarios have been suggested: one is the conventional relativistic outflow with initial Lorentz factor of order of $\Gamma_{0} \gtrsim 10$ and the other is a trans-relativistic outflow with $\Gamma_{0} \simeq 1-2$. Here we compare the high energy gamma-ray afterglow emission from these two different models, taking into account both synchrotron self inverse-Compton scattering (SSC) and the external inverse-Compton scattering due to photons from the cooling supernova or hypernova envelope (SNIC). We find that the conventional relativistic outflow model predicts a relatively high gamma-ray flux from SSC at early times $\left(<10^{4} \mathrm{~s}\right.$ for typical parameters) with a rapidly decaying light curve, while in the trans-relativistic outflow model, one would expect a much flatter light curve of high-energy gamma-ray emission at early times, which could be dominated by both the SSC emission and SNIC emission, depending on the properties of the underlying supernova and the shock parameter $\epsilon_{e}$ and $\epsilon_{B}$. The Fermi Gamma-ray Space Telescope should be able to distinguish between the two models in the future.
\end{abstract}

Subject headings: gamma-rays: burst

\section{INTRODUCTION}

Long duration gamma-ray bursts are generally believed to result from the death of massive stars, and their association with core-collapse supernovae ( $\mathrm{SNe}$ of Type $\mathrm{Ib} / \mathrm{c}$ ) has been observed

\footnotetext{
${ }^{1}$ Department of Astronomy, Nanjing University, Nanjing 210093, China

${ }^{2}$ Institute of Astrophysics, Huazhong Normal University, Wuhan 430079, China

${ }^{3}$ Department of Astronomy and Astrophysics, Pennsylvania State University, University Park, PA 16802, USA

${ }^{4}$ Department of Physics, Pennsylvania State University, University Park, PA 16802, USA

${ }^{5}$ Key Laboratory for Particle Astrophysics, Institute of High Energy Physics, Chinese Academy of Sciences, Beijing 100049, China
} 
over the last decade. The first hint for such a connection came with the discovery of a nearby SN 1998bw in the error circle of GRB 980425 (Galama et al. 1998; Iwamoto et al. 1998) at distance of only about $40 \mathrm{Mpc}$. The isotropic gamma-ray energy release is of the order of only $10^{48} \mathrm{erg}$ (Galama et al. 1998) and the radio afterglow modelling suggests an energy of $10^{49}-10^{50} \mathrm{erg}$ in a mildly relativistic ejecta (Kulkarni et al. 1998; Chevalier \& Li 1999). Recently Swift discovered GRB 060218, which is the second nearest GRB identified so far (Campana et al. 2006; Cusumano et al. 2006; Mirabal \& Halpern 2006; Sakamoto 2006). It is also an under-energetic burst with energy in prompt $\gamma / \mathrm{X}$-rays about $5 \times 10^{49} \mathrm{erg}$ and is associated with SN 2006aj. Another burst, GRB 031203, is a third example of this group (Sazonov et al. 2004).

If one assumes that GRB 060218-like bursts follow the $\log N-\log P$ relationship of high-luminosity GRBs then, as argued by Guetta et al. (2004), no such burst with redshifts $z<0.17$ should be observed by a HETE-like instrument within the next 20 years. Therefore, the unexpected discovery of GRB 060218 may suggest that these objects form a different new class of GRBs from the conventional high-luminosity GRBs (Soderberg et al. 2006; Pian et al. 2006; Cobb et al. 2006; Liang et al. 2006; Guetta \& Della Valle 2007; Dai 2008), although what distinguishes such low-luminosity GRBs (LL-GRBs) from the conventional high-luminosity GRBs (HL-GRBs) remains unknown. Their rate of occurrence may be one order of magnitude higher than that of the typical ones (e.g. Soderberg et al. 2008). They are rarely recorded because such intrinsically dim GRBs can only be detected from relatively short distances with present gamma-ray instruments.

The "compactness problem" of GRBs requires that the outflow of normal GRBs should be highly relativistic with a bulk Lorentz factor of $\Gamma_{0} \gtrsim 100$ (Baring \& Harding 1997; Lithwick \& Sari 2001). The low-luminosity and softer spectra of low-luminosity GRBs relax this constraint on the bulk Lorentz factor. It was suggested that the softer spectrum and low energetics of GRB060218 (or classified as X-ray flash 060218) may indicate a somewhat lower Lorentz factor of the order of 10 (e.g. Mazzali 2006; Fan et al. 2006; Toma et al. 2006).

An alternative possibility is that low-luminosity GRBs are driven by a trans-relativistic outflow with $\Gamma_{0} \simeq 2$ (Waxman 2004; Waxman, Mészáros \& Campana, 2007; Wang, Li, Waxman \& Mészáros 2007; Ando \& Mészáros 2008). The flat light curve of the X-ray afterglow of the nearest GRB, GRB 980425/SN 1998bw, up to 100 days after the burst, has been argued to result from the coasting phase of a mildly relativistic shell with an energy of a few times $10^{49} \mathrm{erg}$ (Waxman 2004). From the thermal energy density in the prompt emission of GRB060218/SN2006aj, Campana et al. (2006) inferred that the shell driving the radiation-dominated shock in GRB 060218/SN 2006aj must be mildly relativistic. This trans-relativistic shock could be driven by the outermost parts of the envelope that get accelerated to a mildly relativistic velocity when the supernova shock accelerates in the density gradient of the envelope of the supernova progenitor (Colgate 1974; Matzner \& McKee 1999; Tan et al. 2001), or it could be due to a choked relativistic jet propagating through 
the progenitor (Wang et al. 2007).

In this paper, we investigate the high energy afterglow emission from low luminosity GRBs for both relativistic and trans-relativistic models and explore whether the Fermi Gamma-ray Space Telescope can distinguish between these two models with future observations of low-luminosity GRBs. Since photons from the underlying supernova are an important seed photon source for inverse-Compton (IC) scattering, we consider both synchrotron self-inverse Compton (SSC) and external IC scattering due to supernova photons (denoted by SNIC hereafter). At early times (within a few days after the burst), a UV-optical SN component was recently detected from SN2006aj and SN2008D (Campana et al. 2006; Soderberg et al. 2008), which has been interpreted as the cooling SN envelope emission after being heated by the radiation-dominated shock 1 (Waxman et al. 2007; Soderberg et al. 2008; Chevalier \& Fransson 2008). In our calculation, we take into account this seed photon source in addition to the late-time supernova emission, which peaks after ten days.

Recently, Ando \& Meszaros (2008) discussed the broadband emission from SSC and SNIC for a trans-relativistic ejecta in a low-luminosity GRB at a particular time- the ejecta deceleration time. Here we study the time evolution of the high-energy gamma-ray emission resulted from such IC processes and consider both the trans-relativistic ejecta and the highly relativistic ejecta scenarios for low-luminosity GRBs.

The paper is organized as follows. First, we describe the dynamics of shock evolution in the two models in $\S 2$. In $\S 3$, we present the formula for the calculation of the inverse-Compton emissivity. For the SNIC emission, we take into account the anisotropic scattering effect. Then we present the results of the spectra and light curves of SSC and SNIC emission for the two different models and explore the detectability of these components by Fermi Large Area Telescope (LAT) in $\S 4$. Finally, we give the conclusions and discussions.

\section{Dynamics and Electron Energy Distribution}

We assume that the two models have the same parameters except for the initial Lorentz factor of the ejecta. For the latter, we adopt nominal values of $\Gamma_{0}=10$ in the conventional relativistic ejecta model and $\Gamma_{0}=2$ in the trans-relativistic ejecta model, respectively. Note that in the conventional relativistic ejecta model, even if $\Gamma_{0} \gg 10$ the dynamics of the blast wave is identical to the case of $\Gamma_{0}=10$ from the time tens of seconds after the burst, because the blast wave has entered

\footnotetext{
${ }^{1}$ Although there is a disagreement on the origin of the early UV-optical emission from SN2006aj, an agreement has been reached for that of SN2008D (Waxman et al. 2007; Soderberg et al. 2008; Chevalier \& Fransson 2008).
} 
the Blandford-McKee self-similar phase since then.

We consider a spherical GRB ejecta carrying a total energy of $E=10^{50} E_{50}$ erg expanding into a surrounding wind medium with density profile $n=K r^{-2}$, where $K \equiv \dot{M} /\left(4 \pi m_{p} v_{\mathrm{w}}\right)=3 \times 10^{35} \mathrm{~cm}^{-1} \dot{m}$ with $\dot{m} \equiv\left(\dot{M} / 10^{-5} M_{\odot} \mathrm{yr}^{-1}\right) /\left(\mathrm{v}_{\mathrm{w}} / 10^{3} \mathrm{kms}^{-1}\right)$. As the circumburst medium is swept up by the blast wave, the total kinetic energy of the fireball is (Panaitescu et al. 1998)

$$
E_{\mathrm{k}}=(\gamma-1)\left(m_{\mathrm{ej}}+m\right) c^{2}+\gamma U^{\prime}
$$

where $\gamma$ is the bulk Lorentz factor of the shell, $m_{\mathrm{ej}}$ the ejecta mass, $m$ is the mass of the sweptup medium, and the comoving internal energy $U^{\prime}$ can be expressed by $U^{\prime}=(\gamma-1) m c^{2}$, which is suitable for both ultrarelativistic and Newtonian shocks (Huang et al. 1999). Hereafter superscript prime represents that the quantities are measured in the comoving frame of the shell. As usual, we assume that the magnetic field and the electrons have a fraction $\epsilon_{B}$ and $\epsilon_{e}$ of the internal energy, respectively. Following Eq. (11), the differential dynamic equation can be derived as (Huang et al. 2000)

$$
\frac{d \gamma}{d m}=-\frac{\gamma^{2}-1}{m_{\mathrm{ej}}+2 \gamma m}
$$

which describes the overall evolution of the shell from relativistic phase to non-relativistic phase. The initial value of $\gamma$ is $\Gamma_{0}=E /\left(m_{\mathrm{ej}} c^{2}\right)$. To obtain the time-dependence of $\gamma$ one makes use of

$$
\begin{gathered}
\frac{d m}{d R}=4 \pi R^{2} n m_{\mathrm{p}}, \\
\frac{d R}{d t}=\beta c \gamma\left(\gamma+\sqrt{\gamma^{2}-1}\right),
\end{gathered}
$$

where $t$ is the observer time, $R$ and $\beta=\sqrt{1-\gamma^{-2}}$ are the radius and velocity of the shell, respectively.

Solving Eqs. 2, 3, and 4, three dynamic phases can be found: (i) Coasting phase. The shell does not decelerate significantly until it arrives at the deceleration radius, $R_{\mathrm{dec}}=E /\left(4 \pi K \Gamma_{0}^{2} m_{\mathrm{p}} c^{2}\right)$, where the mass of the swept-up medium $m$ is comparable to $m_{\mathrm{ej}} / \Gamma_{0}$ (Sari \& Piran 1995). The corresponding deceleration time can be calculated from $t_{\mathrm{dec}} \simeq R_{\mathrm{dec}} /\left(2 \Gamma_{0}^{2} c\right)$. For representative parameter values $E_{50}=1$ and $\dot{m}=1, R_{\mathrm{dec}} \simeq 4.4 \times 10^{15} \mathrm{~cm}$ and $t_{\mathrm{dec}} \simeq 1.8 \times 10^{4} \mathrm{~s}$ for a trans-relativistic ejecta with $\Gamma_{0}=2$, while $R_{\mathrm{dec}} \simeq 1.8 \times 10^{14} \mathrm{~cm}$ and $t_{\mathrm{dec}} \simeq 29 \mathrm{~s}$ for a conventional highly relativistic ejecta with $\Gamma_{0}=10$; (ii) Blandford \& McKee self-similar phase $\left(t>t_{\mathrm{dec}}\right.$ and $\left.\gamma \gtrsim 2\right)$, where $\gamma \propto t^{-1 / 4}$ and $R \propto t^{1 / 2}$; (iii) Non-relativistic phase $(\gamma \rightarrow 1)$, where $\beta \propto t^{-1 / 3}$ and $R \propto t^{2 / 3}$, i.e., the Sedov-von Neumann-Taylor solution applies (Zel'dovich \& Raizer 2002, p.93, Waxman, 2004).

In the absence of radiation losses, the energy distribution of shock-accelerated electrons behind the shock is usually assumed to be a power-law as $d N_{e} / d \gamma_{e} \propto \gamma_{e}^{-p}$. As the electrons are cooled 
by synchrotron and IC radiation, the electron energy distribution becomes a broken power-law, given by

(1) for $\gamma_{e, \mathrm{c}} \leq \gamma_{e, \mathrm{~m}}$,

$$
\frac{d N_{e}}{d \gamma_{e}} \propto \begin{cases}\gamma_{e}^{-2}, & \gamma_{e, \mathrm{c}} \leq \gamma_{e} \leq \gamma_{e, \mathrm{~m}} \\ \gamma_{e}^{-p-1}, & \gamma_{e, \mathrm{~m}}<\gamma_{e} \leq \gamma_{e, \max }\end{cases}
$$

(2) for $\gamma_{e, \mathrm{~m}}<\gamma_{e, \mathrm{c}} \leq \gamma_{e, \max }$,

$$
\frac{d N_{e}}{d \gamma_{e}} \propto \begin{cases}\gamma_{e}^{-p}, & \gamma_{e, \mathrm{~m}} \leq \gamma_{e} \leq \gamma_{e, \mathrm{c}} \\ \gamma_{e}^{-p-1}, & \gamma_{e, \mathrm{c}}<\gamma_{e} \leq \gamma_{e, \max }\end{cases}
$$

which are normalized by the total number of the electrons solved from the dynamic equations. The minimum, cooling, and maximum Lorentz factors of electrons are, respectively, given by

$$
\begin{aligned}
\gamma_{e, \mathrm{~m}} & =\epsilon_{e} \frac{p-2}{p-1} \frac{m_{\mathrm{p}}}{m_{e}}(\gamma-1)=92 f_{p 1} \epsilon_{e,-0.5}(\gamma-1) \\
\gamma_{e, \mathrm{c}}= & \frac{6 \pi m_{e} c}{(1+Y) \sigma_{T} B^{\prime 2}\left(\gamma+\sqrt{\gamma^{2}-1}\right) t} \\
& \simeq \frac{1.7 \times 10^{3} R_{15}^{2}}{\epsilon_{B,-3} \dot{m} t_{4} Y\left(\gamma+\sqrt{\gamma^{2}-1}\right) \gamma(\gamma-1)} \\
\gamma_{e, \max }= & \sqrt{\frac{6 \pi q_{e}}{\sigma_{T} B^{\prime}(1+Y)}} \\
& \simeq 4.5 \times 10^{7} R_{15} \gamma^{-1 / 4}(\gamma-1)^{-1 / 4} Y^{-1 / 2}
\end{aligned}
$$

where $f_{p 1}=6(p-2) /(p-1), B^{\prime}$ is the comoving magnetic field strength and $Y$ is the Compton parameter that is defined as the ratio of the IC luminosity (including SSC and SNIC) to the synchrotron luminosity2.

\section{Inverse-Compton Emission}

The accelerated electrons can be cooled by synchrotron radiation and inverse Compton (IC) scattering of seed photons (including synchrotron photons and blackbody photons emitted by the supernova). Since the IC emissivity on the basis of the Thomson cross section is inaccurate for high-energy $\gamma$-rays, we use the full Klein-Nishina cross section instead. Once the electron distribution and the flux of seed photons $\left(f_{\nu_{\mathrm{s}}^{\prime}}^{\prime}\right)$ (the distribution of which is isotropic) are known, the IC

\footnotetext{
${ }^{2}$ At very late times, when $\gamma_{e, \mathrm{~m}}$ decreases to be close to a few, $\gamma_{e, \mathrm{~m}}=\epsilon_{e} \frac{p-2}{p-1} \frac{m_{\mathrm{p}}}{m_{e}}(\gamma-1)+1$ is used (Huang \& Cheng 2003).
} 
emissivity (at frequency $\nu^{\prime}$ ) of electrons can be calculated by(Blumenthal \& Gould 1970; Yu et al. 2007)

$$
\varepsilon_{\text {iso }}^{\prime \mathrm{IC}}\left(\nu^{\prime}\right)=3 \sigma_{\mathrm{T}} \int_{\gamma_{e, \min }}^{\gamma_{e, \max }} d \gamma_{e} \frac{d N_{e}}{d \gamma_{e}} \int_{\nu_{\mathrm{s}, \min }^{\prime}}^{\infty} d \nu_{\mathrm{s}}^{\prime} \frac{\nu^{\prime} f_{\nu_{\mathrm{s}}^{\prime}}^{\prime}}{4 \gamma_{e} \nu_{\mathrm{s}}^{\prime 2}} g(x, y),
$$

where $\gamma_{\mathrm{e}, \min }=\max \left[\gamma_{\mathrm{e}, \mathrm{c}}, \gamma_{\mathrm{e}, \mathrm{m}}, h \nu^{\prime} /\left(m_{\mathrm{e}} c^{2}\right)\right], \nu_{\mathrm{s}, \min }^{\prime}=\nu^{\prime} m_{\mathrm{e}} c^{2} / 4\left[\gamma_{\mathrm{e}}\left(\gamma_{\mathrm{e}} m_{\mathrm{e}} c^{2}-h \nu^{\prime}\right)\right], x=4 \gamma_{\mathrm{e}} h \nu_{\mathrm{s}}^{\prime} / m_{\mathrm{e}} c^{2}, y=$ $h \nu^{\prime} /\left[x\left(\gamma_{\mathrm{e}} m_{\mathrm{e}} c^{2}-h \nu^{\prime}\right)\right]$, and

$$
g(x, y)=2 y \ln y+(1+2 y)(1-y)+\frac{1}{2} \frac{x^{2} y^{2}}{(1+x y)}(1-y) .
$$

In our case, because the radius of the supernova photosphere is much smaller than that of the GRB shock, supernova seed photons can be regarded as a point photon source locating at the center in the comoving frame of the GRB shock. These soft photons from supernova impinge on the shock region basically along the radial direction in the rest frame of the shock, so the scatterings between these photons and the isotropically-distributed electrons in the shock are anisotropic. For a photon beam penetrating into the shock region where the electrons are moving isotropically, the inverse Compton scattering emissivity of the radiation scattered at an angle $\theta_{\mathrm{SC}}$ relative to the direction of the photon beam in the shock comoving frame is (Aharonian \& Atoyan 1981, Brunetti 2000, Fan et. al. 2008):

$$
\begin{aligned}
& \varepsilon^{\mathrm{AIC}}\left(\nu^{\prime}, \cos \theta_{\mathrm{SC}}\right)=\frac{3 \sigma_{\mathrm{T}} c}{16 \pi} \int_{\gamma_{e, \min }}^{\gamma_{e, \max }} d \gamma_{\mathrm{e}} \frac{d N_{\mathrm{e}}}{d \gamma_{e}} \\
& \int_{\nu_{\mathrm{s}, \min }^{\prime}}^{\infty} \frac{f_{\nu_{\mathrm{s}}^{\prime}}^{\prime \prime \mathrm{S}} d \nu_{\mathrm{s}}^{\prime}}{\gamma_{\mathrm{e}}^{2} \nu_{\mathrm{s}}^{\prime}}\left[1+\frac{\xi^{2}}{2(1-\xi)}-\frac{2 \xi}{b_{\theta}(1-\xi)}+\frac{2 \xi^{2}}{b_{\theta}^{2}(1-\xi)^{2}}\right]
\end{aligned}
$$

where $\xi \equiv h \nu^{\prime} /\left(\gamma_{e} m_{e} c^{2}\right), b_{\theta}=2\left(1-\cos \theta_{\mathrm{SC}}\right) \gamma_{e} h \nu_{\mathrm{s}}^{\prime} /\left(m_{e} c^{2}\right)$ and $h \nu_{\mathrm{s}}^{\prime} \ll h \nu^{\prime} \ll \gamma_{e} m_{e} c^{2} b_{\theta} /\left(1+b_{\theta}\right)$. On integration over $\theta_{\mathrm{SC}}$ for whole solid angle (i.e. in the case that the photon distribution is also isotropic), Eq.(13) reduces to Eq.(11), i.e. the usual isotropic inverse Compton scattering emissivity. In the observer frame, the angle $\theta$ between the injecting photons and scattered photons relates with the angle $\theta_{\mathrm{SC}}$ in the comoving frame by $\cos \theta_{\mathrm{SC}}=(\cos \theta-\beta) /(1-\beta \cos \theta)$, where $\beta$ is the velocity of the GRB shock.

The observed SSC and SNIC flux densities at a frequency $\nu$ are given respectively by (e.g. Huang et al. 2000, Yu et al. 2007)

$$
F_{\nu}^{\mathrm{SSC}}=\int_{0}^{\pi} \frac{\varepsilon_{\text {iso }}^{\prime \mathrm{IC}}(\nu / D)}{4 \pi D_{\mathrm{L}}^{2}} D^{3} \sin \theta d \theta
$$

and

$$
F_{\nu}^{\mathrm{SNIC}}=\int_{0}^{\pi} \frac{\varepsilon^{\mathrm{AIC}}\left(\nu / D, \cos \theta_{\mathrm{SC}}\right)}{4 \pi D_{\mathrm{L}}^{2}} D^{3} \sin \theta d \theta
$$

where $D_{\mathrm{L}}$ is the luminosity distance and $D \equiv[\gamma(1-\beta \cos \theta)]^{-1}$ is the Doppler factor. Note that $\cos \theta_{\mathrm{SC}}$ in Eq.(15) can be transformed to $\cos \theta$ through $\cos \theta_{\mathrm{SC}}=(\cos \theta-\beta) /(1-\beta \cos \theta)$. 


\subsection{Seed Photons from the Supernova}

For seed photons from the supernova, we consider the contributions from two components. One is the early thermal UV-optical emission from the cooling supernova envelope after being heated by the radiation-dominated shock (Waxman et al. 2007; Chevalier \& Fransson 2008). Such UV-optical emission has been observed recently by Swift UVOT from SN2006aj (Campana et al. 2006) and SN2008D (Soderberg et al. 2008). Another component is the supernova optical emission at later time, powered by the radioactive elements synthesized in supernovae.

The characterization of the emission from the cooling supernova envelope is expressed as follows, which is correct at $t \gtrsim 10^{2}$ s (Waxman, Mészáros, \& Campana 2007):

$$
\begin{aligned}
& R_{\mathrm{ph}}(t)=3.2 \times 10^{14} \frac{E_{\text {snej }, 51}^{0.4}}{\left(M_{\text {snej }} / M_{\odot}\right)^{0.3}} t_{\text {day }}^{0.8} \mathrm{~cm}, \\
& T_{\mathrm{ph}}(t)=2.2 \frac{E_{\text {snej }, 51}^{0.02}}{\left(M_{\text {snej }} / M_{\odot}\right)^{0.03}} R_{0,12}^{1 / 4} t_{\text {day }}^{-0.5} \mathrm{eV}, \\
& L_{\mathrm{ph}}(t)=4 \pi R_{\mathrm{ph}}(t)^{2} \sigma T_{\mathrm{ph}}(t)^{4}
\end{aligned}
$$

where $R_{\mathrm{ph}}(t)$ and $T_{\mathrm{ph}}(t)$ are the radius and temperature of the envelope, $L_{\mathrm{ph}}(t)$ is the luminosity from the photosphere of the cooling envelope, $M_{\text {snej }}$ and $E_{\text {snej }}$ are the supernova ejecta mass and energy, and $R_{0}$ is the initial stellar radius of the $\mathrm{SN}$ progenitor. We take the following values for supernovae like SN2006aj and SN2008D: $E_{\text {snej, } 51}=E_{\text {snej }} /\left(10^{51} \mathrm{erg}\right)=2, M_{\text {snej }}=2 M_{\odot}$ and $R_{0,12}=R_{0} /\left(10^{12} \mathrm{~cm}\right)=$ 0.3 (Mazzali et al. 2006; Soderberg et al. 2008). For hypernovae such as SN1998bw associated with GRB980425, modelling of the SN optical emission gives a larger kinetic energy and ejecta mass such as $E_{\text {snej,51 }} \simeq 22$ and $M_{\text {snej }}=6 M_{\odot}$ for SN1998bw (Woosley et al. 1999).

The luminosity of the late component is rising before $\sim 10$ days and then decaying exponentially. For SN2008D, it is found that the rising is roughly in proportion to $t^{1.6}$ (Soderberg et. al. 2008), so we can describe the luminosity as

$$
L_{\mathrm{rad}}(t)= \begin{cases}3 \times 10^{42}\left(\frac{t}{10 \text { days }}\right)^{1.6} \mathrm{erg} \mathrm{s}^{-1}, & t<10 \text { days } \\ 3 \times 10^{42} \exp \left(1-\frac{t}{10 \text { days }}\right) \text { erg s }^{-1}, & t \geq 10 \text { days }\end{cases}
$$

We assumed that the radiation temperature is approximately constant, $T_{\text {rad }} \simeq 1 \mathrm{eV}$. The bolometric luminosity of the cooling supernova envelop is dominant over that of the late component before $t=7.8 \times 10^{5} \mathrm{~s}$.

Through the calculation of the shock dynamic evolution, the radii of the GRB shock are $R=1.9 \times 10^{14} \mathrm{~cm}$ at $t=10^{3} \mathrm{~s}$, and $R=1.5 \times 10^{15} \mathrm{~cm}$ at $t=10^{4} \mathrm{~s}$ for the trans-relativistic ejecta model, and for the conventional ejecta model $R=1.0 \times 10^{15} \mathrm{~cm}$ at $t=10^{3} \mathrm{~s}$ and $R=4.1 \times 10^{15} \mathrm{~cm}$ 
at $t=10^{4} \mathrm{~s}$. According to Eq 16 , the shell radii for supernova are $9.6 \times 10^{12} \mathrm{~cm}$ at $t=10^{3} \mathrm{~s}$ and $6.1 \times 10^{13} \mathrm{~cm}$ at $t=10^{4} \mathrm{~s}$, and the one for hypernova are $1.8 \times 10^{13} \mathrm{~cm}$ at $t=10^{3} \mathrm{~s}$ and $1.1 \times 10^{14} \mathrm{~cm}$ at $t=10^{4} \mathrm{~s}$. Consequently the shell radius is less than ten percents of the GRB ejecta height, so it is reasonable to approximate that supernova photons come from a point source at the center and impinge onto the electrons in the shock from behind.

\subsection{Compton Parameter $Y$}

Following Moderski, Sikora \& Bulik (2000) and Sari \& Esin (2001), we define the Compton parameter $Y$ as

$$
Y \equiv \frac{L_{\mathrm{IC}}}{L_{\mathrm{SYN}}}=\frac{u_{\gamma}^{\prime}}{u_{B}^{\prime}}=\frac{u_{\mathrm{SYN}}^{\prime}+f_{\mathrm{a}} u_{\mathrm{SN}}^{\prime}}{u_{B}^{\prime}}
$$

where

$$
\begin{aligned}
u_{\mathrm{SN}}^{\prime}= & \gamma^{-2} \frac{L_{\mathrm{SN}}}{4 \pi c R^{2}} \\
& \simeq 65 \mathrm{erg} \mathrm{cm}^{-3} t_{4}^{-0.4} R_{15}^{-2} \gamma^{-2} \\
u_{\mathrm{SYN}}^{\prime}= & \frac{\eta \epsilon_{e} u^{\prime}}{(1+Y)} \\
& \simeq 5.4 \times 10^{2} \mathrm{erg} \mathrm{cm}^{-3} \epsilon_{e,-0.5} \dot{m} R_{15}^{-2} \gamma(\gamma-1) \eta Y^{-1} \\
u_{B}^{\prime}= & \epsilon_{B} u^{\prime} \\
& \simeq 1.8 \mathrm{erg} \mathrm{cm}{ }^{-3} \epsilon_{B,-3} \dot{m} R_{15}^{-2} \gamma(\gamma-1),
\end{aligned}
$$

are, respectively, the comoving energy densities of the blackbody supernova seed photons, synchrotron seed photons and magnetic fields, and $f_{\mathrm{a}}$ is the factor accounting for the suppression of the photon energy density due to the anisotropic inverse Compton scattering effect $\left(f_{\mathrm{a}}=1\right.$ corresponds to the isotropic scattering case). Here $L_{\mathrm{SN}}=L_{\mathrm{ph}}+L_{\mathrm{rad}}$, which is dominated by the luminosity of the cooling envelop $L_{\mathrm{ph}}, u^{\prime}$ is the comoving internal energy density, $\eta=\eta_{\mathrm{rad}} \eta_{\mathrm{KN}}$ is the radiation efficiency where $\eta_{\mathrm{rad}}$ is the fraction that the electron's energy radiated, and $\eta_{\mathrm{KN}}$ is the fraction of synchrotron photons below the KN limit frequency (Nakar 2007). For slow cooling, $\eta_{\mathrm{rad}}=\left(\gamma_{e, \mathrm{c}} / \gamma_{e, \mathrm{~m}}\right)^{2-p}($ Moderski, Sikora \& Bulik 2000), and

$$
\eta_{\mathrm{KN}}= \begin{cases}0, & \nu_{\mathrm{KN}}^{\prime}\left(\gamma_{e, \mathrm{c}}\right) \leq \nu_{\mathrm{m}}^{\prime} \\ \left(\frac{\nu_{\mathrm{KN}}^{\prime}\left(\gamma_{e, \mathrm{c}}\right)}{\nu_{\mathrm{c}}^{\prime}}\right)^{(3-p) / 2}, & \nu_{\mathrm{m}}^{\prime}<\nu_{\mathrm{KN}}^{\prime}\left(\gamma_{e, \mathrm{c}}\right)<\nu_{\mathrm{c}}^{\prime} \\ 1, & \nu_{\mathrm{c}}^{\prime} \leq \nu_{\mathrm{KN}}^{\prime}\left(\gamma_{e, \mathrm{c}}\right)\end{cases}
$$

For fast cooling, $\eta_{\mathrm{rad}}=1$ and

$$
\eta_{\mathrm{KN}}= \begin{cases}0, & \nu_{\mathrm{KN}}^{\prime}\left(\gamma_{e, \mathrm{~m}}\right) \leq \nu_{\mathrm{c}}^{\prime} \\ \left(\frac{\nu_{\mathrm{KN}}^{\prime}\left(\gamma_{e, \mathrm{~m}}\right)}{\nu_{\mathrm{m}}^{\prime}}\right)^{1 / 2}, & \nu_{\mathrm{c}}^{\prime}<\nu_{\mathrm{KN}}^{\prime}\left(\gamma_{e, \mathrm{~m}}\right)<\nu_{\mathrm{m}}^{\prime} \\ 1, & \nu_{\mathrm{m}}^{\prime} \leq \nu_{\mathrm{KN}}^{\prime}\left(\gamma_{e, \mathrm{~m}}\right)\end{cases}
$$


Solving eq. (20), we get

$$
Y=\frac{1}{2}\left[\sqrt{4 \frac{\eta \epsilon_{e}}{\epsilon_{B}}+\left(1+\frac{f_{\mathrm{a}} u_{\mathrm{SN}}^{\prime}}{\epsilon_{B} u^{\prime}}\right)^{2}}+\left(\frac{f_{\mathrm{a}} u_{\mathrm{SN}}^{\prime}}{\epsilon_{B} u^{\prime}}-1\right)\right] .
$$

Roughly, the above expression can be simplified in three limiting cases as follows

$$
Y= \begin{cases}f_{\mathrm{a}} u_{\mathrm{SN}}^{\prime} /\left(\epsilon_{B} u^{\prime}\right), & f_{\mathrm{a}} u_{\mathrm{SN}}^{\prime} \gg u_{\mathrm{SYN}}^{\prime} \\ \sqrt{\eta \epsilon_{e} / \epsilon_{B}}, & u_{B}^{\prime} \ll f_{\mathrm{a}} u_{\mathrm{SN}}^{\prime} \ll u_{\mathrm{SYN}}^{\prime} \\ \left(\sqrt{4 \eta \epsilon_{e} / \epsilon_{B}+1}-1\right) / 2, & f_{\mathrm{a}} u_{\mathrm{SN}}^{\prime} \ll \min \left[u_{\mathrm{SYN}}^{\prime}, u_{B}^{\prime}\right]\end{cases}
$$

In the latter two cases, $Y$ can be treated as a constant when the electrons are in the fast-cooling regime and $\nu_{\mathrm{m}}^{\prime} \leq \nu_{\mathrm{KN}}^{\prime}\left(\gamma_{e, \mathrm{~m}}\right)$ with $\eta=1$.

\subsection{Pair Production Opacity For High Energy Photons}

High-energy gamma-rays can be attenuated due to interaction with low-energy photons through the pair production effect. We consider the pair production opacity in the shock frame due to the absorption by low-energy photons, which include thermal photons from the supernova, synchrotron photons, SSC photons and SNIC photons. A high energy photon of energy $E_{\gamma, 1}^{\prime}$ in the shock frame will annihilate with a low energy photon of $E_{\gamma, 2}^{\prime}$, provided that $E_{\gamma, 1}^{\prime} E_{\gamma, 2}^{\prime}\left(1-\cos \theta_{12}\right) \geq 2\left(m_{e} c^{2}\right)^{2}$, where $\theta_{12}$ is the collision angle of the two annihilation photons. The pair creation cross section is given by

$$
\sigma\left(E_{\gamma, 1}^{\prime}, E_{\gamma, 2}^{\prime}\right)=\frac{1}{2} \pi r_{0}^{2}\left(1-\tilde{\beta}^{2}\right)\left[\left(3-\tilde{\beta}^{4}\right) \ln \frac{1+\tilde{\beta}}{1-\tilde{\beta}}-2 \tilde{\beta}\left(2-\tilde{\beta}^{2}\right)\right],
$$

where $\left.\tilde{\beta} \equiv v / c=\sqrt{1-2\left(m_{e} c^{2}\right)^{2} /\left[E_{\gamma, 1}^{\prime} E_{\gamma, 2}^{\prime}\left(1-\cos \theta_{12}\right)\right.}\right]$ is the velocity of electrons in the center-ofmass frame (Heitler 1954, Stecker, De Jager, \& Salamon, 1992) and $r_{0}=e^{2} /\left(m_{e} c^{2}\right)$ is the classic electron radius. By using the photon distribution in the shock frame, we obtain the optical depth for high-energy photons of energy,

$$
\tau\left(E_{\gamma, 1}^{\prime}\right)=\int_{E_{t h r}^{\prime}}^{\infty} \sigma\left(E_{\gamma, 1}^{\prime}, E_{\gamma, 2}^{\prime}\right) n_{\gamma}\left(E_{\gamma, 2}^{\prime}\right) \frac{R}{\eta_{\mathrm{R}}} d E_{\gamma, 2}^{\prime},
$$

where $\eta_{\mathrm{R}}$ is the shock compressed ratio, which is $\eta_{\mathrm{R}}=4 \gamma+3$, and the threshold energy of low energy photons is $E_{\mathrm{thr}}^{\prime}=2\left(m_{e} c^{2}\right)^{2} / E_{\gamma, 1}^{\prime}\left(1-\cos \theta_{12}\right)$. In the calculation, we estimate the cutoff energy conservatively by assuming that colliding photons are moving isotropically in the shock frame. Such a treatment may overestimate the pair-production opacity because in reality the supernova seed photons move anisotropically (i.e. moving outward in radial direction as seen by high-energy photons emitted from the shock at much larger radii). 
The result of the cutoff energy in the observer frame $E_{\gamma, \text { cut }}=\gamma E_{\gamma}^{\prime}(\tau=1)$ is given in Figure 1 . Since the luminosity and peak energy of the supernova envelope emission decreases with time in general and the shock radius increases with time, the cutoff energy of the high energy spectrum increases with time, which is clearly seen in Fig. 1. From this figure, we can see that the cutoff energy is above $1 \mathrm{GeV}$ after the starting time of our calculation $\left(10^{2.5} \mathrm{~s}\right)$, so we can calculate the light curves at energy $\sim 1 \mathrm{GeV}$ without considering the opacity. Since the cutoff energy is large enough, it does not affect the detectability of Fermi LAT, whose sensitive energy band is $20 \mathrm{MeV} \sim$ $300 \mathrm{GeV}$.

\section{Results}

\subsection{Spectra and Light Curves: Numerical Results}

We first compare the spectra of different IC components for the two models. The spectra at $t=10^{3} \mathrm{~s}$ are shown in Figure 2, including the synchrotron emission, the thermal emission from the supernova or hypernova, the synchrotron self-Compton emission and the SNIC emission, for parameters $\epsilon_{e}=0.1, \epsilon_{B}=0.001, p=2.2, E=10^{50} \mathrm{erg}$ and burst distance $D_{L}=100 \mathrm{Mpc}$. From the spectra we can see that for the supernova case the SSC emission dominates over the SNIC emission at energies from $1 \mathrm{MeV}$ to $100 \mathrm{GeV}$ for conventional relativistic ejecta model, and for trans-relativistic ejecta model the SSC emission is higher than SNIC emission and the two components are both important at high energies below the cutoff energy. For the hypernova case the SSC emission is dominant in the conventional relativistic ejecta model while the SNIC emission is dominant in the trans-relativistic ejecta model at the high energy band. This is because in the case of hypernovae and $\Gamma_{0}=2$, the energy density of hupernova photons $u_{\mathrm{HN}}^{\prime}$ (multiplied by the suppressed factor $f_{\mathrm{a}}$ due to the anisotropic scattering) is significantly higher than the synchrotron radiation density $u_{\mathrm{SYN}}^{\prime}$.

Figure 3 shows light curves of SSC and SNIC afterglow emission at $h \nu=1 \mathrm{GeV}$ in the two models for $E=10^{50} \mathrm{ergs}, p=2.2$ and four different sets of parameters of $\epsilon_{e}$ and $\epsilon_{B}$ for the supernova case. In the conventional relativistic model with $\Gamma_{0}=10$, a sharp decay phase of a $\mathrm{GeV}$ afterglow is produced during the early hours, which is mildly dominated by the SSC emission, and a slightly flatter decay phase dominated by SNIC emission takes over at late times $t>10^{6} \mathrm{~s}$. On the other hand, for the trans-relativistic ejecta model with $\Gamma_{0}=2$, a plateau of SSC emission, due to the presence of a coasting phase in the ejecta dynamic, dominates in the early time, which transits to a faster decay at later time and SNIC emission become dominant after the time $10^{5} \sim 10^{6} \mathrm{~s}$.

Figure 4 show light curves for the hypernovae case. Light curves in the conventional relativistic ejecta model are similar with those for the supernova case except that SNIC emission become 
dominant at earlier time; for the trans-relativistic ejecta model, SNIC emission is always dominated with parameters $\epsilon_{e}=0.1$ and $\epsilon_{B}=0.001$, a plateau is seen in the early time, which transits to a faster decay at later time.

The two models also predict different flux levels at high-energies. In early hours, the total flux from the conventional relativistic ejecta model is more than one order of magnitude higher than that in the trans-relativistic ejecta model. This can be explained by the different amount of energy in shocked electrons. For the conventional relativistic ejecta model, the ejecta has been decelerated at this time and a great part of its energy has been converted into shocked electrons, while in the trans-relativistic ejecta model, only a small fraction of the ejecta kinetic energy has been converted to shocked electrons at this early time. A lower amount of energy in shocked electrons results in a lower flux level in the trans-relativistic ejecta model.

In addition, a comparison among four panels in Figure 3 and Figure 4 indicates that the flux decreases as $\epsilon_{e}$ decreases, which is obvious since the energy of radiating electrons $E_{e} \propto \epsilon_{e}$. As $\epsilon_{B}$ decreases, the SSC flux changes little and the SNIC flux increases for the case that SSC emission is dominant at early time. This can be understood from the following analysis. Since the SSC

emission dominates and $f_{\mathrm{a}} u_{\mathrm{SN}}^{\prime} \gg u_{B}^{\prime}$ at the early times, $Y \simeq \sqrt{\eta \epsilon_{e} / \epsilon_{B}} \propto \epsilon_{B}^{-\frac{1}{2}} \epsilon_{e}^{\frac{1}{2}}$. The SSC and SNIC flux at $\nu>\nu_{\min }>\nu_{\mathrm{c}}$ scale as

$$
\nu F_{\nu}^{\mathrm{SSC}}=\nu F_{\max }^{\mathrm{SSC}}\left(\frac{\nu_{\min }^{\mathrm{SSC}}}{\nu_{c}^{\mathrm{SSC}}}\right)^{-\frac{1}{2}}\left(\frac{\nu}{\nu_{\min }^{\mathrm{SSC}}}\right)^{-\frac{p}{2}} \propto \epsilon_{B}^{\frac{p}{4}-\frac{1}{2}} \epsilon_{e}^{2 p-3}
$$

and

$$
\nu F_{\nu}^{\mathrm{SNIC}}=\nu F_{\max }^{\mathrm{SNIC}}\left(\frac{\nu_{\min }^{\mathrm{SNIC}}}{\nu_{c}^{\mathrm{SNIC}}}\right)^{-\frac{1}{2}}\left(\frac{\nu}{\nu_{\min }^{\mathrm{SNIC}}}\right)^{-\frac{p}{2}} \propto \epsilon_{B}^{-\frac{1}{2}} \epsilon_{e}^{p-\frac{3}{2}}
$$

\subsection{The effect of the anisotropic scattering on the SNIC emission}

The incoming supernova photons are anisotropic as seen by the isotropically distributed electrons in the GRB shock, so the IC scatterings are anisotropic. In order to see how the anisotropic inverse-Compton scattering (AIC) affects the SNIC flux, we compare the light curves of the SNIC emission obtained by using the isotropic scattering formula Eq.11 and using the AIC scattering formula Eq.13 in Figure 5. The thinner lines show the SNIC light curves obtained using the usual isotropic scattering formula, while the thicker ones correspond to the calculations with the AIC scattering effect taken into account. One can see that the flux of the SNIC emission with the AIC effect correction is reduced by a factor of about $\sim 0.4$ compared to the isotropic scattering case. This is consistent with the calculation result obtained by Fan \& Piran (2006), who studied the anisotropic inverse Compton scattering between inner optical/X-ray flare photons and electrons in the outer GRB forward shock. 
Fig. 5 shows that the AIC effect suppresses the SNIC flux only slightly. The anisotropic photon distribution results in more head-on scatterings, i.e. the photon beam scatter preferentially with those electrons that move in the direction antiparallel to the photon beam, so one can expect that the scattered IC emission power has a maximum at $\theta_{\mathrm{SC}}=\pi$ and goes to zero for small scattering angles (e.g. Brunetti 2000). The photons scattered into the angles $0 \lesssim \theta_{\text {SC }} \lesssim \pi / 2$ relative to the shock moving direction in the shock comoving frame will fall into the cone of angle $1 / \Gamma$ in the observer frame, according to the transformation formula $\cos \theta_{\mathrm{SC}}=(\cos \theta-\beta) /(1-\beta \cos \theta)$. Therefore the AIC scatterings decrease the IC emission in the $1 / \Gamma$ cone along the direction of the photon beam, but meanwhile they enhance the emission at larger angles (about half of the emission falling into angles between $1 / \Gamma$ and $2 / \Gamma$, see Wang \& Mészáros 2006). For a spherical outflow as we consider here, the IC emission after integration over angles should have the same flux in every direction in the observer frame, with a flux level only slightly reduced comparable to the isotropic scattering case.

\subsection{Analytical Light Curves}

As a comparison, we derive here approximate analytical expressions for afterglow light curves, which provide an explanation for the physical origin of the behavior. Since the anisotropic SNIC emission are depressed by a factor of 0.4 , which is almost constant, relative to the isotropic seed photons case, we can consider the isotropic seed photons case for the approximate analytic treatment of the afterglow light curves. The blackbody photons from the supernova can be approximated as mono-energetic photons with $h \nu_{\mathrm{SN}}=2.7 \mathrm{KT}_{\mathrm{SN}}$. Thus, similar to the description for synchrotron emission of a single electron (Sari et al. 1998), the radiation power and characteristic frequencies of SNIC from a single electron scattering supernova photons in the observer frame can be described by

$$
P\left(\gamma_{e}\right)=\frac{4}{3} \sigma_{T} c \gamma^{2} \gamma_{e}^{2} \frac{L_{\mathrm{SN}}}{\gamma^{2} \pi R^{2} c}
$$

and

$$
\nu\left(\gamma_{e}\right)=2 \gamma \gamma_{e}^{2} \nu_{\mathrm{SN}} / \gamma=2 \gamma_{e}^{2} \nu_{\mathrm{SN}},
$$

respectively. Similar to the analysis in Sari and Esin (2001), the maximum flux of the SNIC spectrum is

$$
F_{\max }^{\mathrm{SNIC}}=\frac{N_{e}}{4 \pi D_{\mathrm{L}}^{2}} \frac{P\left(\gamma_{e}\right)}{\nu\left(\gamma_{e}\right)}
$$

Characteristic SNIC frequencies are

$$
\nu_{\mathrm{min}}^{\mathrm{SNIC}}=2 \gamma_{e, \mathrm{~m}}^{2} \nu_{\mathrm{SN}}
$$


and

$$
\nu_{c}^{\mathrm{SNIC}}=2 \gamma_{e, \mathrm{c}}^{2} \nu_{\mathrm{SN}}
$$

respectively.

By adopting the broken power-law approximation for the IC spectral component ( Sari \& Esin 2001) and the dynamics of the shock discussed in $\S 2$, one can derive the analytic light curves in an approximate way.

\subsubsection{Light Curves In The Conventional Relativistic Ejecta Model}

In the conventional relativistic ejecta model, at time $t_{\mathrm{dec}}<t<t_{\text {tran }}$, where $t_{\text {tran }}$ is defined as the transition time when $\gamma_{e, \mathrm{~m}}=\gamma_{e, \mathrm{c}}$, we have $\eta=1$ and $u_{B}^{\prime} \ll f_{\mathrm{a}} u_{\mathrm{SN}}^{\prime} \ll u_{\mathrm{SYN}}^{\prime}$, so $Y \simeq \sqrt{\eta \epsilon_{e} / \epsilon_{B}} \propto t^{0}$. The shock dynamic follows the Blandford \& McKee self-similar solution in the wind medium, i.e. $R \propto t^{\frac{1}{2}}$ and $\gamma \propto t^{-\frac{1}{4}}$. Then we can obtain the evolution of the break frequencies of the SSC and SNIC spectral components and their peak flux in the following way:

$$
\begin{gathered}
\nu_{\min }^{\mathrm{SSC}}=2 \gamma_{e, \mathrm{~m}}^{2} \nu_{\min } \propto t^{-2}, \nu_{\mathrm{c}}^{\mathrm{SSC}}=2 \gamma_{e, \mathrm{c}}^{2} \nu_{\mathrm{c}} \propto t^{2}, \\
F_{\nu, \max }^{\mathrm{SSC}}=\frac{\sigma_{T} N_{e}}{4 \pi R^{2}} F_{\max }^{\mathrm{SYN}} \propto t^{-1} .
\end{gathered}
$$

and

$$
\nu_{\min }^{\mathrm{SNIC}} \propto t^{-1}, \nu_{\mathrm{c}}^{\mathrm{SNIC}} \propto t, F_{\nu, \max }^{\mathrm{SNIC}} \propto t^{-0.4}
$$

where $\nu_{\mathrm{SN}} \propto T_{\mathrm{SN}} \propto t^{-1 / 2}$ has been used for the cooling envelope emission (see Eq. 13). The SSC and SNIC flux at an observed frequency $\nu$ higher than characteristic frequencies vary as $\nu F_{\nu}^{\mathrm{SSC}} \propto t^{-p+1}$ and $\nu F_{\nu}^{\mathrm{SNIC}} \propto t^{-\frac{p}{2}+0.6}$ respectively for $t<t_{\text {tran }}$.

At $t>t_{\mathrm{SNIC}}$, where $t_{\mathrm{SNIC}}$ is the time when $f_{\mathrm{a}} u_{\mathrm{SN}}^{\prime}=u_{\mathrm{SYN}}^{\prime}$, we take $Y \simeq f_{\mathrm{a}} u_{\mathrm{SN}}^{\prime} /\left(\epsilon_{B} u^{\prime}\right)$ because the SNIC emission becomes dominated. At such time, the shock is likely to enter the non-relativistic phase, so we take the Sedov-von Neumann-Taylor solutions $R \propto t^{\frac{2}{3}}$ and $\beta \propto t^{-\frac{1}{3}}$, which induce that $Y \propto t^{\frac{2}{3}-0.4}$. So the minimum and cooling Lorentz factors of electrons vary as $\gamma_{e, \mathrm{~m}} \propto \beta^{2} \propto t^{-\frac{2}{3}}$ and $\gamma_{e, \mathrm{c}} \propto \beta^{-2} R^{2} t^{-1} Y^{-1} \propto t^{0.4+\frac{1}{3}}$. Thus, the break frequencies of the SSC and SNIC spectral components and their peak fluxes evolve with time in the following way:

$$
\nu_{\min }^{\mathrm{SSC}} \propto t^{-\frac{11}{3}}, \nu_{\mathrm{c}}^{\mathrm{SSC}} \propto t^{\frac{1}{3}+1.6}, F_{\nu, \max }^{\mathrm{SSC}} \propto t^{-1},
$$

and

$$
\nu_{\min }^{\mathrm{SNIC}} \propto t^{-\frac{11}{6}}, \nu_{\mathrm{c}}^{\mathrm{SNIC}} \propto t^{\frac{1}{6}+0.8}, F_{\nu, \max }^{\mathrm{SNIC}} \propto t^{-\frac{2}{3}+0.1} .
$$

Then the SSC and SNIC fluxes at high energy $\nu$ vary as $\nu F_{\nu}^{\mathrm{SSC}} \propto t^{1.8-\frac{11}{6} p}$ and $\nu F_{\nu}^{\mathrm{SNIC}} \propto t^{\frac{5}{6}-\frac{11}{12} p}$ at $t>t_{\mathrm{SNIC}}$. 
To summarize, the temporal evolution of the SSC and SNIC afterglow emission at high energies are

$$
\nu F_{\nu}^{\mathrm{SSC}} \propto \begin{cases}t^{-1.2} & t \lesssim t_{\mathrm{tran}} \\ t^{-2.2} & t>t_{\mathrm{SNIC}}\end{cases}
$$

and

$$
\nu F_{\nu}^{\mathrm{SNIC}} \propto \begin{cases}t^{-0.5} & t \lesssim t_{\mathrm{tran}} \\ t^{-1.2} & t>t_{\mathrm{SNIC}}\end{cases}
$$

in the two asymptotic phases for $p=2.2$.

\subsubsection{Light Curves In The Trans-relativistic Ejecta Model}

In the trans-relativistic ejecta model, one would expect a much flatter light curve of highenergy gamma-ray emission at early times, which could be dominated by both the SSC emission and SNIC emission, depending on the properties of the underlying supernova and the shock parameter $\epsilon_{e}$ and $\epsilon_{B}$. For the supernova case, the SSC emission is dominant before the deceleration time and the transition time for most parameters, while for the hypernova case, the SSC emission is dominant at earlier time with $\epsilon_{e}=0.3$ and $\epsilon_{B}=0.01$ and the SNIC emission is always dominant for $\epsilon_{e}=0.1$ and $\epsilon_{B}=0.001$.

For cases where SSC emission dominated in trans-relativistic ejecta model, we have $\eta=1$ in fast cooling regime and $u_{B}^{\prime} \ll f_{\mathrm{a}} u_{\mathrm{SN}}^{\prime} \ll u_{\mathrm{SYN}}^{\prime}$, so $Y \simeq \sqrt{\eta \epsilon_{e} / \epsilon_{B}} \propto t^{0}$. Since $t<t_{\mathrm{dec}}$, we adopt the approximation $R \propto t, \gamma \propto t^{0}$. So the SSC and SNIC flux vary as $\nu F_{\nu}^{\mathrm{SSC}} \propto t^{1-\frac{p}{2}}$ and $\nu F_{\nu}^{\mathrm{SNIC}} \propto t^{0.1-\frac{p}{4}}$ respectively at $t<\min \left(t_{\mathrm{dec}}, t_{\mathrm{tran}}\right)$. At later time when $t>t_{\mathrm{dec}}$ and $\gamma \rightarrow 1$, the SNIC emission become dominant, the evolution of light curves is the same as that in conventional relativistic ejecta model, i.e. the SSC and SNIC flux vary as $\nu F_{\nu}^{\mathrm{SSC}} \propto t^{1.8-\frac{11}{6} p}$ and $\nu F_{\nu}^{\mathrm{SNIC}} \propto t^{\frac{5}{6}-\frac{11}{12} p}$ at $t>t_{\mathrm{dec}}$. Therefore, for the supernova case and the hypernova case with early dominated SSC emission, the temporal evolution of the SSC and SNIC emission at high frequency $\nu$ for $p=2.2$ are given by

$$
\nu F_{\nu}^{\mathrm{SSC}} \propto \begin{cases}t^{-0.1} & t \lesssim \min \left(t_{\mathrm{dec}}, t_{\mathrm{tran}}\right) \\ t^{-2.2} & t \gg t_{\mathrm{dec}}\end{cases}
$$

and

$$
\nu F_{\nu}^{\mathrm{SNIC}} \propto \begin{cases}t^{-0.45} & t \lesssim \min \left(t_{\mathrm{dec}}, t_{\mathrm{tran}}\right) \\ t^{-1.2} & t \gg t_{\mathrm{dec}}\end{cases}
$$

For cases where SNIC emission dominated in trans-relativistic ejecta model, We have $f_{\mathrm{a}} u_{\mathrm{SN}}^{\prime} \gg$ $u_{\mathrm{SYN}}^{\prime}$, so $Y \simeq f_{\mathrm{a}} u_{\mathrm{SN}}^{\prime} /\left(\epsilon_{B} u^{\prime}\right)$. At the time $t<t_{\mathrm{dec}}$, we adopt the approximation $R \propto t, \gamma \propto t^{0}$ to yield $Y \propto t^{-0.4}$. So the SSC and SNIC flux vary as $\nu F_{\nu}^{\mathrm{SSC}} \propto t^{1.8-\frac{p}{2}}$ and $\nu F_{\nu}^{\mathrm{SNIC}} \propto t^{0.5-\frac{p}{4}}$ respectively at 
$t<\min \left(t_{\mathrm{dec}}, t_{\mathrm{tran}}\right)$. At later time when $t>t_{\mathrm{dec}}$ and $\gamma \rightarrow 1$, the evolution of light curves is the same as that in conventional relativistic ejecta model, i.e. the SSC and SNIC flux vary as $\nu F_{\nu}^{\mathrm{SSC}} \propto t^{1.8-\frac{11}{6} p}$ and $\nu F_{\nu}^{\mathrm{SNIC}} \propto t^{\frac{5}{6}-\frac{11}{12} p}$ at $t>t_{\mathrm{dec}}$. Therefore, for the supernova case and the hypernova case with early dominated SSC emission, the temporal evolution of the SSC and SNIC emission at high frequency $\nu$ for $p=2.2$ are given by

$$
\nu F_{\nu}^{\mathrm{SSC}} \propto \begin{cases}t^{0.7} & t \lesssim \min \left(t_{\mathrm{dec}}, t_{\mathrm{tran}}\right) \\ t^{-2.2} & t \gg t_{\mathrm{dec}}\end{cases}
$$

and

$$
\nu F_{\nu}^{\mathrm{SNIC}} \propto \begin{cases}t^{-0.05} & t \lesssim \min \left(t_{\mathrm{dec}}, t_{\mathrm{tran}}\right) \\ t^{-1.2} & t \gg t_{\mathrm{dec}}\end{cases}
$$

\subsection{Detectability by the Fermi LAT}

We explore here whether Fermi LAT can detect the high energy gamma-ray emission from low luminosity GRBs in the two models considered above. Following Zhang \& Mészáros (2001), Gou \& Mészáros (2007) and Yu, Liu \& Dai (2007), the fluence threshold for long-duration observations is $F_{\mathrm{thr}}=\left[\phi_{0}\left(t / t_{\mathrm{eff}}\right)^{1 / 2}\right] E_{\mathrm{ph}} t_{\mathrm{eff}}$ which is in proportional to $t^{1 / 2}$ due to the limitation by the background, where we take the average energy of the detected photons as $E_{\mathrm{ph}}=400 \mathrm{MeV}$ and the effective time as $t_{\text {eff }}=0.5 \mathrm{yr} . \phi_{0}$ is the integral sensitivity above $100 \mathrm{MeV}$ for LAT for a steady source after a year sky survey, which is $\phi_{0} \sim 3 \times 10^{-9} \mathrm{phscm}^{-2} \mathrm{~s}^{-1}$ (atwood et. al. 2009) and is improved by a factor of 3 by keeping the GRB position at the center of the LAT field of view as long as possible (Gou \& Mészáros 2007). For short-time observation, the fluence threshold is calculated by $F_{\mathrm{thr}}=5 E_{\mathrm{ph}} / A_{\mathrm{eff}}$ under the assumption that at least 5 photons are collected. Taking the effective area $A_{\text {eff }}=6000 \mathrm{~cm}^{2}$, we can obtain the fluence threshold of Fermi LAT,

$$
F_{\mathrm{thr}}= \begin{cases}5.3 \times 10^{-7} \mathrm{erg} \mathrm{cm}^{-2}, & t \leq 4.4 \times 10^{4} \mathrm{~s}, \\ 2.5 \times 10^{-9} t^{1 / 2} \mathrm{erg} \mathrm{cm}^{-2}, & t>4.4 \times 10^{4} \mathrm{~s} .\end{cases}
$$

With this fluence threshold, the detectability of high energy emission (with supernova seed photons luminosity given above and a total energy $E=10^{50} \mathrm{erg}$ ) by the Fermi LAT is shown in Figure 6. The time-integrated fluence shown in the plot is defined as an integration of the flux density $\left(F_{\nu}\right)$ over the Fermi LAT energy band $[20 \mathrm{MeV}, 300 \mathrm{GeV}]$ and the time interval $[0.5 t, t]$ as used in Gou \& Meszaros (2007), which is $\int_{0.5 t}^{t} \int_{\nu_{1}}^{\nu_{2}} F_{\nu} d \nu d t$.

For $\epsilon_{e}=0.3$ and other representative parameter values, high-energy gamma-ray emission in the conventional relativistic ejecta model stays detectable up to $\sim 10^{6}$ s, while the high-energy gamma-ray emission in the trans-relativistic ejecta model can only be detected in a short period around $10^{4.5} \mathrm{~s}$. For a lower value such as $\epsilon_{e}=0.1$, the high-energy gamma-ray emission can still 
be detected in the conventional relativistic ejecta model, while it becomes undetectable for the trans-relativistic ejecta model.

In order to compare with earlier results in Ando \& Mészáros (2008), we increase the total energy to $E=2 \times 10^{50} \mathrm{erg}$, which yields a kinetic energy $E_{\mathrm{k}}=\left(\Gamma_{0}-1\right) / \Gamma_{0} E=10^{50} \mathrm{erg}$ in the transrelativistic ejecta model, the same as that used in Ando \& Mészáros (2008). This will increase the fluence by a factor of 2 . We also increase the SN luminosity from that of a normal SN (shown in $\S$ 3.1) to SN1998bw-like hypernovae. In Figure 7, we show the detectability of high energy emission by Fermi LAT in this case. By comparing the flux of light curves between the supernova case and the hypernova case, which is shown in Fig. 3] and Fig. 4, we can see that increasing the SN luminosity can hardly enhance the total IC flux for the two models. Our IC flux is still lower than that obtained by Ando \& Mészáros (2008). The main difference between our calculation and that of Ando \& Mészáros (2008) is the different minimum Lorentz factors used in the calculations. Ando \& Mészáros (2008) may overestimate the minimum Lorentz factor of electrons by taking $\gamma_{e, \mathrm{~m}}=\epsilon_{e}\left(m_{p} / m_{e}\right) \gamma$, which is a factor of $(p-1) /(p-2)$ larger than ours. From the formula describing the high energy flux $\nu F_{\nu}^{\mathrm{SNIC}} \propto \nu_{\min , \mathrm{SNIC}}^{(p-1) / 2} \propto \gamma_{e, \mathrm{~m}}^{p-1}$, one expects that the flux is increased by a factor of $\left(\frac{p-1}{p-2}\right)^{p-1}$, which is about 8 for $p=2.2$.

\section{Discussions and Conclusions}

The external stellar wind provides a source of Thomson opacity to scatter the supernova emission, thus a quasi-isotropic, back-scattered SN radiation field is present. Let's study whether this component is important. For a GRB shock locating at radius $R$ and moving with a Lorentz factor $\gamma$, the Thomson scattering optical depth of the wind is $\tau_{\mathrm{w}}=\sigma_{T} R n=\sigma_{T} K R^{-1}=2.0 \times 10^{-4} \dot{m} R_{15}^{-1}$. The scattered SN energy density by the wind in the comoving frame of the blast wave is $u_{\mathrm{SN}}^{\prime \mathrm{w}}=$ $\left(L_{S N} / 4 \pi c R^{2}\right) \tau_{\mathrm{w}} \gamma^{2}$ due to the relativistic boosting effect. On the other hand, the energy density of the supernova photons impinging the shock from behind is $u_{\mathrm{SN}}^{\prime}=\gamma^{-2}\left(L_{S N} / 4 \pi c R^{2}\right)$ (see Eq.21). The ratio between these two energy densities is $u_{\mathrm{SN}}^{\prime \mathrm{w}} / u_{\mathrm{SN}}^{\prime}=2.0 \times 10^{-4} \dot{m} R_{15}^{-1} \gamma^{4}$. For the trans-relativistic ejecta model with $\Gamma_{0}=2$, the radius of the GRB ejecta is $R=1.9 \times 10^{14} \mathrm{~cm}$ at $t=10^{3} \mathrm{~s}$, so $u_{\mathrm{SN}}^{\prime \mathrm{W}} \ll u_{\mathrm{SN}}^{\prime}$ for typical wind parameters. For the conventional relativistic ejecta model, the Lorentz factor and radius of the GRB ejecta are respectively $\gamma \sim 4$ and $R=1.0 \times 10^{15} \mathrm{~cm}$ at $t=10^{3} \mathrm{~s}$, so we also have $u_{\mathrm{SN}}^{\prime \mathrm{W}} \ll u_{\mathrm{SN}}^{\prime}$. This means that the wind-scattered supernova radiation field is subdominant compared to the direct impinging supernova photon field and hence we neglect its contribution to the high-energy gamma-ray emission.

Our estimate of the pair-production opacity for high-energy photons in $\S 3.3$ is based on the common assumption that the colliding photons are isotropic in the rest frame. However, as we have shown above, the low-energy photons from the supernova essentially move radially outward 
before colliding with high-energy photons (e.g. Wang, Li \& Mészáros 2006). Therefore, the collision process between the high-energy photons and soft supernova photons is anisotropic, which would decrease the pair-production opacity. This will be the subject of a more detailed future calculation and would be useful to explore whether $\mathrm{TeV}$ photons can escape from the source, which is important for checking the detectability by ground-based Cherenkov detectors such as Magic, VERITAS, Milago, HESS, ARGO etc. Additionally, besides the high-energy gamma-ray emission discussed in this work, the high-energy neutrino emission arising from $p \gamma$ interactions between shock-accelerated protons and photons from the supernova may also provide a constraint on the model for low-luminosity GRBs (e.g. Yu et al. 2008).

Trans-relativistic ejecta may also exist in the usual high luminosity long GRBs, besides in low luminosity ones, since accelerating shocks are expected to accompany the supernova. Berger et al. (2003) and Sheth et al. (2003) found that the radio and optical afterglow indicates a low velocity component more than 1.5days after the explosion in GRB030329/SN2003dh. However, since the highly relativistic ejecta is much more energetic than the trans-relativistic component, high-energy gamma-ray emission from the latter component could easily remain hidden.

In summary, we have calculated the spectra and light curves of the high-energy gamma-ray afterglow emission from low luminosity GRBs for the two main models in the literature, i.e. the trans-relativistic ejecta model $\left(\Gamma_{0} \simeq 2\right)$ and the conventional highly relativistic ejecta model ( $\Gamma_{0} \gtrsim 10$ ), considering both synchrotron self inverse-Compton (SSC) and the external inverseCompton due to photons from the underlying supernova/hypernova. Our analysis takes into account a full Klein-Nishina cross section for inverse Compton scatterings, the anisotropic scattering of supernova photons and the opacity for high energy photons due to annihilation with low-energy photons (mainly from the supernova/hypernova).

We find that for the supernova case the conventional relativistic outflow model predicts a relatively high gamma-ray flux from SSC at early times $\left(<10^{5}-10^{6} \mathrm{~s}\right.$ for typical parameters) with a rapidly decreasing flux, while in the trans-relativistic outflow model, a much flatter light curve of high-energy gamma-ray emission dominated by the SSC emission, is expected at early times. For the hypernova case, the SSC emission also dominates and decays sharply at early time in the conventional relativistic ejecta model, while for the trans-relativistic ejecta model, both the SSC emission and the SNIC emission could be dominant at early time, depending on the shock parameters $\epsilon_{e}$ and $\epsilon_{B}$. The main difference between these two models arises from their different initial Lorentz factors, which induces a different dynamical evolution of the shock at early times. As a result, different observational features arise, such as different light curve shapes, different flux levels and different dominant components (as detailed in $\S$ 4.1). As shown in $\S$ 4.4, highenergy gamma-ray emission can be detected in both models as long as $\epsilon_{e}$ is large enough, although detection from the conventional relativistic ejecta is much easier. Thus, with future high energy 
gamma-ray observations by Fermi LAT, one can expect to be able to distinguish between the two models based on the above differences in observational features.

We would like to thank Z. G. Dai, Y. F. Huang and D. M. Wei for useful discussions. This work is supported by the National Natural Science Foundation of China under grants 10973008 and 10403002, the National Basic Research Program of China (973 program) under grants No. 2009CB824800, the Foundation for the Authors of National Excellent Doctoral Dissertations of China, the Qing Lan Project, and NASA grant NNX 08AL40G. 


\section{REFERENCES}

Aharonian, F. A., \& Atoyan, A. M. 1981, Ap\&SS, 79, 321

Ando, S., \& Mészáros, P. 2008, ApJ, 689, 351

Atwood, W. B., et al. 2009, ApJ, 697, 1071

Baring, M. G., \& Harding, A. K. 1997, ApJ, 491, 663

Berger, E., Kulkarni, S. R., Frail, D. A., \& Soderberg, A. M. 2003, ApJ, 599, 408

Blandford, R. D., \& McKee, C. F. 1976, Physics of Fluids, 19, 1130

Blumenthal, G. R., \& Gould, R. J. 1970, Reviews of Modern Physics, 42, 237

Brunetti, G. 2000, Astroparticle Physics, 13, 107

Campana, S., et al. 2006, Nature, 442, 1008

Chevalier, R. A., \& Fransson, C. 2008, ApJ, 683, L135

Chevalier, R. A., \& Li, Z.-Y. 1999, ApJ, 520, L29

Cobb, B. E., Bailyn, C. D., van Dokkum, P. G., \& Natarajan, P. 2006, ApJ, 645, L113

Colgate, S. A. 1974, ApJ, 187, 333

Cusumano, G., Moretti, A., Tagliaferri, G., Kennea, J., \& Burrows,D. 2006, GRB Coordinates Network, 4786, 1

Dai, X. Y. 2008, ApJ, submitted, arXiv:0812.4466

Fan, Y.-Z., Piran, T., \& Xu, D. 2006, Journal of Cosmology and Astro-Particle Physics, 9, 13

Fan, Y.-Z., Piran, T., Narayan, R., \& Wei, D.-M. 2008, MNRAS, 384, 1483

Fan, Y., \& Piran, T. 2006, MNRAS, 370, L24

Galama, T. J., et al. 1998, Nature, 395, 670

Gou, L.-J., \& Mészáros, P. 2007, ApJ, 668, 392

Guetta, D., Perna, R., Stella, L., \& Vietri, M. 2004, ApJ, 615, L73

Guetta, D. \& Della Valle, M., 2007, ApJ, 657, L73 
Heitler, W. 1954, International Series of Monographs on Physics, Oxford: Clarendon, 1954, 3rd ed.,

Huang, Y. F., Dai, Z. G. \& Lu, T. 1999, MNRAS, 309, 513

Huang, Y. F., Gou, L. J., Dai, Z. G., \& Lu, T. 2000, ApJ, 543, 90

Huang, Y. F. \& Cheng, K. S., 2003, MNRAS, 341, 263

Iwamoto, K., et al. 1998, Nature, 395, 672

Kulkarni, S. R., et al. 1998, Nature, 395, 663

Liang, E.-W., Zhang, B.-B., Stamatikos, M., Zhang, B., Norris, J., Gehrels, N., Zhang, J., \& Dai, Z. G. 2006, ApJ, 653, L81

Lithwick, Y., \& Sari, R. 2001, ApJ, 555, 540

Matzner, C. D., \& McKee, C. F. 1999, ApJ, 510, 379

Mazzali, P. A., et al. 2006, Nature, 442, 1018

Mirabal, N., Halpern, J. P., An, D., Thorstensen, J. R., \& Terndrup, D. M. 2006, ApJ, 643, L99

Moderski, R., Sikora, M., \& Bulik, T. 2000, ApJ, 529, 151

Nakar, E. 2007, Phys. Rep., 442, 166

Panaitescu, A., Meszaros, P., \& Rees, M. J. 1998, ApJ, 503, 314

Pian, E., et al. 2006, Nature, 442, 1011

Sakamoto, T., et al. 2006, GRB Coordinates Network, 4822, 1

Sari, R., \& Esin, A. A. 2001, ApJ, 548, 787

Sari, R., \& Piran, T. 1995, ApJ, 455, L143

Sazonov, S. Y.; Lutovinov, A. A.; Sunyaev, R. A. 2004; Nature, 430, 646

Sheth, K., Frail, D. A., White, S., Das, M., Bertoldi, F., Walter, F., Kulkarni, S. R., \& Berger, E. 2003, ApJ, 595, L33

Soderberg, A. M., et al. 2006, Nature, 442, 1014

Soderberg, A. M., et al. 2008, Nature, 454, 246 
Stecker, F. W., de Jager, O. C., \& Salamon, M. H. 1992, ApJ, 390, L49

Tan, J. C., Matzner, C. D., \& McKee, C. F. 2001, ApJ, 551, 946

Toma, K., Ioka, K., Yamazaki, R., \& Nakamura, T. 2006, ApJ, 640, L139

Wang, X.-Y., Li, Z., Waxman, E., \& Mészáros, P. 2007, ApJ, 664, 1026

Wang, X.-Y., Li, Z. \& Mészáros, P. 2006, ApJ, 641, L89

Wang, X.-Y. \& Mészáros, P. 2006, ApJ, 643, L95

Waxman, E. 2004, ApJ, 602, 886

Waxman, E., Mészáros, P., \& Campana, S. 2007, ApJ, 667, 351

Woosley, S. E., Eastman, R. G., \& Schmidt, B. P. 1999, ApJ, 516, 788

Yu, Y. W., Liu, X. W., \& Dai, Z. G. 2007, ApJ, 671, 637

Yu, Y. W., Dai, Z. G., \& Zheng X. P., 2008, MNRAS, 385, 1461

Zeld́ovich, Ya. B., \& Raizer, Yu. P. 2002, Physics of Shockwaves and High Temperature Hydrodynamic Phenomena (Mineola: Dover)

Zhang, B., \& Mészáros, P. 2001, ApJ, 552, L35 


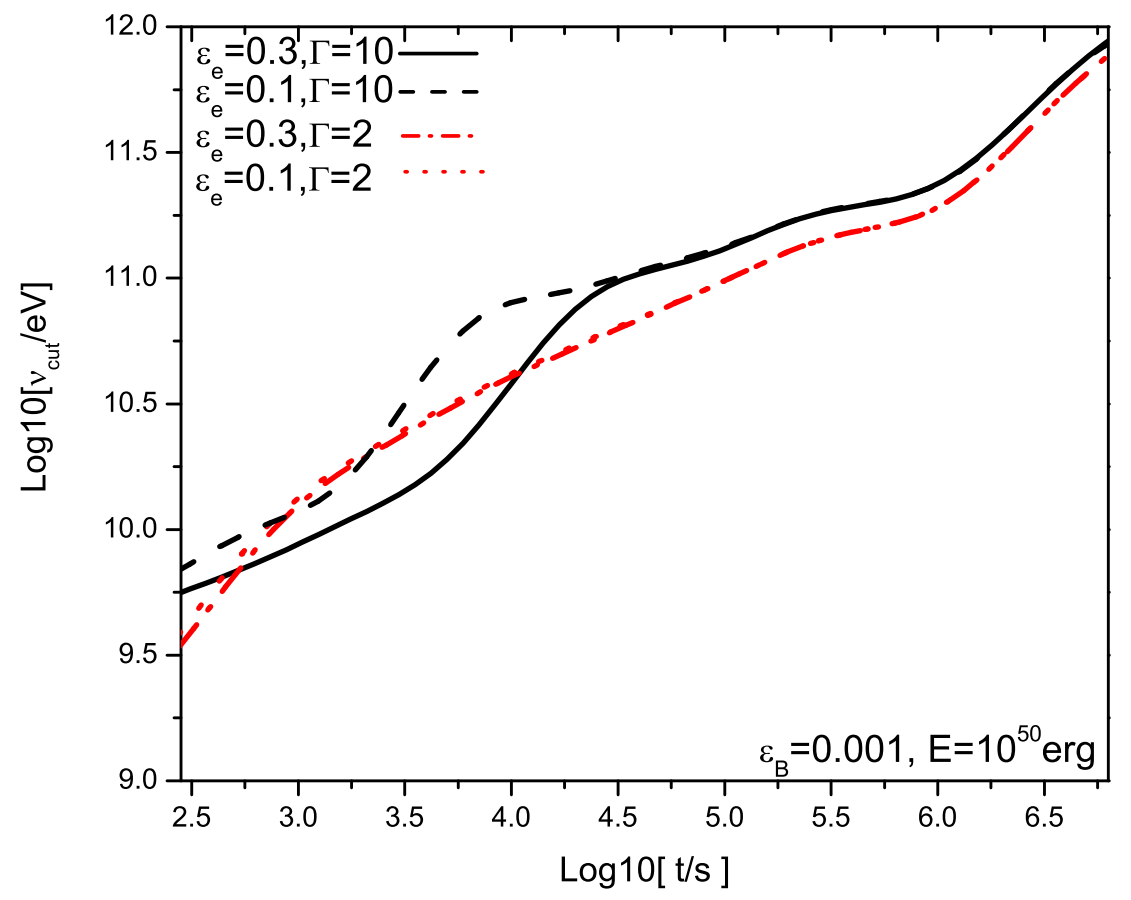

Fig. 1. - The high-energy cutoff due to annihilation with low-energy photons at different times in the two models for low-luminosity GRBs, with $\epsilon_{B}=0.001, p=2.2, \dot{m}=1, E=10^{50} \mathrm{erg}$ and different parameters of $\epsilon_{e}$. The solid lines and dashed lines show the cutoff energy in the conventional relativistic ejecta model with $\epsilon_{e}=0.3$ and $\epsilon_{e}=0.1$, respectively. The dash-dotted and dotted lines show the cutoff energy in the trans-relativistic ejecta model with $\epsilon_{e}=0.3$ and $\epsilon_{e}=0.1$, respectively. 


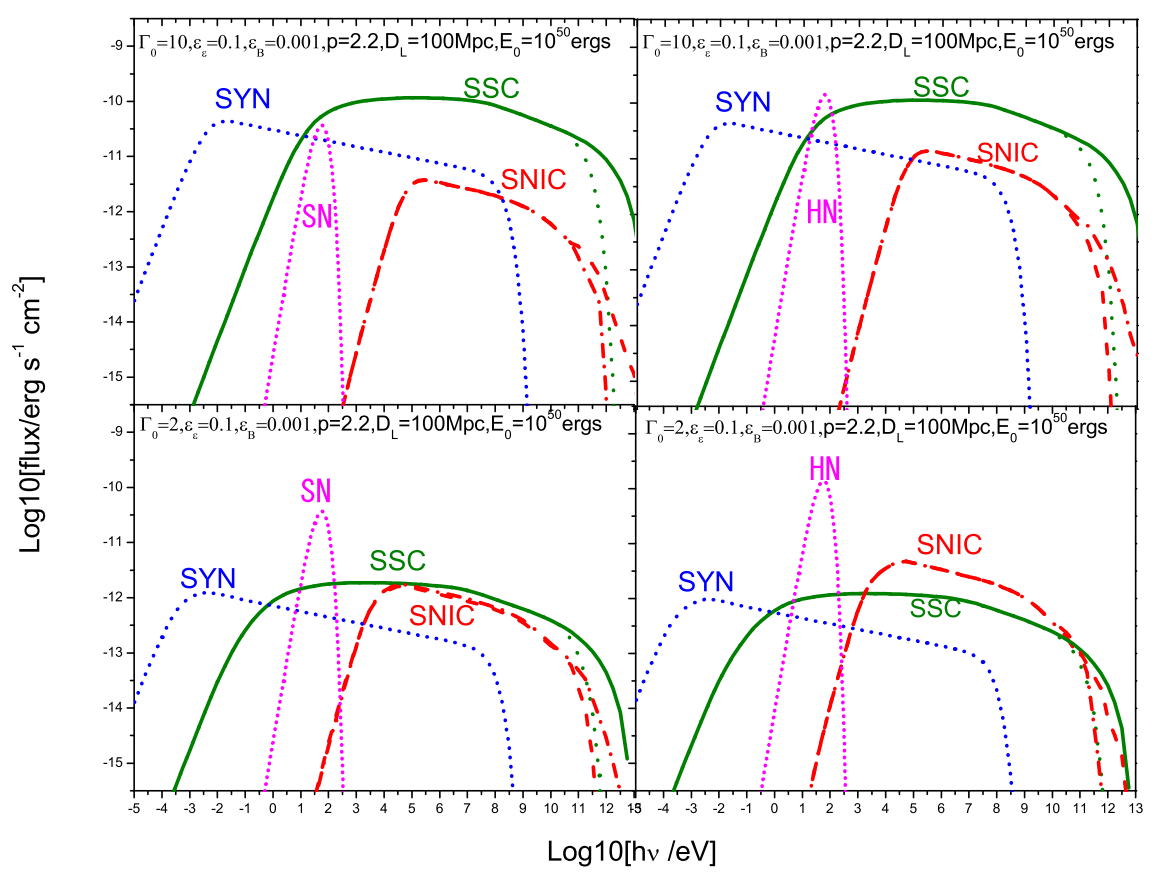

Fig. 2.- The spectra of SNIC, SSC, synchrotron emission and supernova/hypernova photons in the two models at times $t=10^{3} \mathrm{~s}$ for the parameters $\epsilon_{e}=0.1, \epsilon_{e}=0.001, p=2.2$ and $E=10^{50} \mathrm{erg}$. The left panels show the supernova case, the right ones show the hypernova case, the top ones denote the conventional relativistic ejecta model and the bottom ones denote the trans-relativistic ejecta model. The dotted and dash dotted lines show the spectra of SSC and SNIC with the annihilation effect taken into account, while the solid lines and dashed lines show the spcetra of SSC and SNIC emission without considering this effect. The short dashed lines denote the spectra of synchrotron emission and the short dotted lines denote the blackbody spectrum from the supernova/hypernova. 


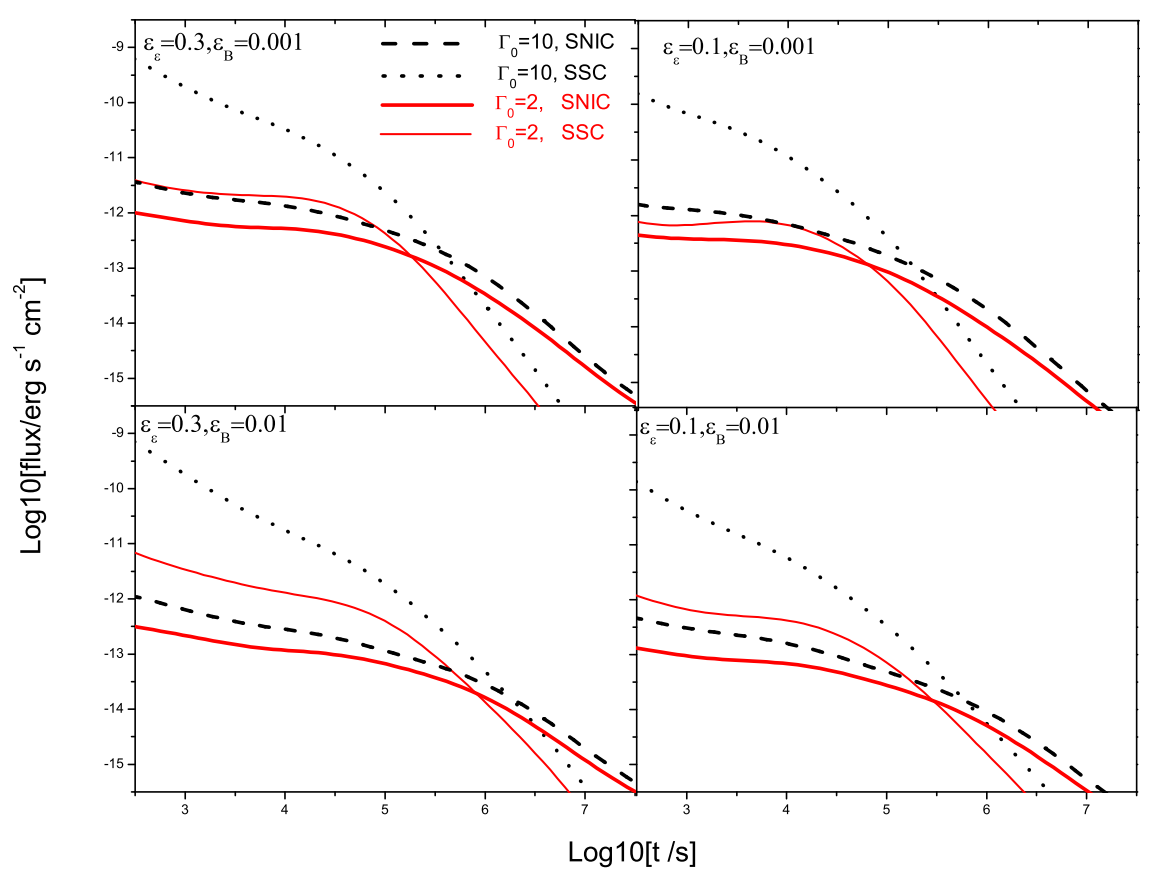

Fig. 3.- Light curves of the SNIC and SSC emission at energy $h \nu=1 \mathrm{GeV}$ in the two models for a normal supernova case and for $E=10^{50} \mathrm{erg}$ and different values for $\epsilon_{e}$ and $\epsilon_{B}$. The thicker and thinner solid lines represent the SNIC and SSC emission in the trans-relativistic ejecta model, and the dashed and the dotted lines represent the SNIC and SSC emission in the conventional relativistic ejecta model. 


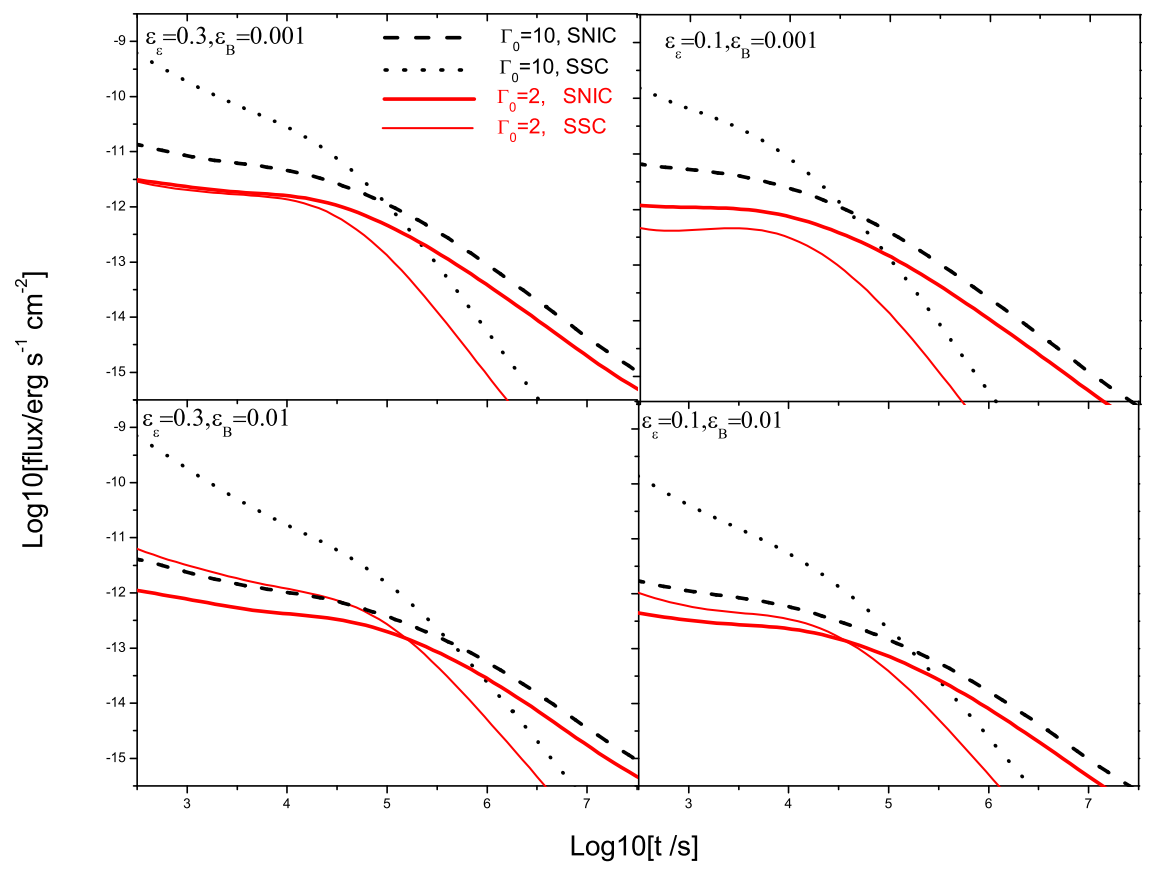

Fig. 4.- Same as Fig.3, but for the hypernova case. The thicker and thinner solid lines represent the SNIC and SSC emission in the trans-relativistic ejecta model, and the dashed and the dotted lines represent the SNIC and SSC emission in the conventional relativistic ejecta model. 


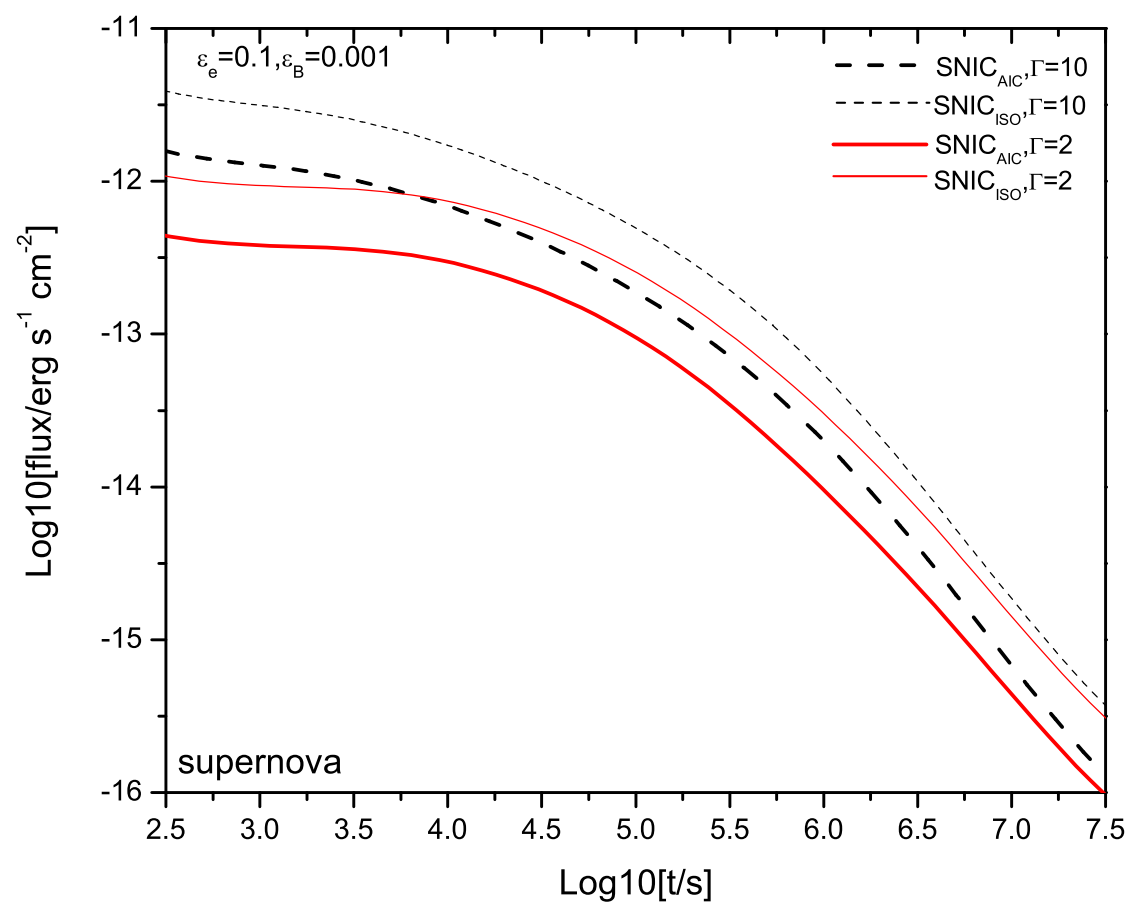

Fig. 5.- Comparison of the light curves of the SNIC emission at $h \nu=1 \mathrm{GeV}$ with (the thicker lines) and without (the thinner lines) the anisotropic scattering effect correction in the two models of low-luminosity GRBs. The solid lines represent the SNIC emission in the trans-relativistic ejecta model, and the dashed lines represent the SNIC emission in the conventional relativistic ejecta model. 


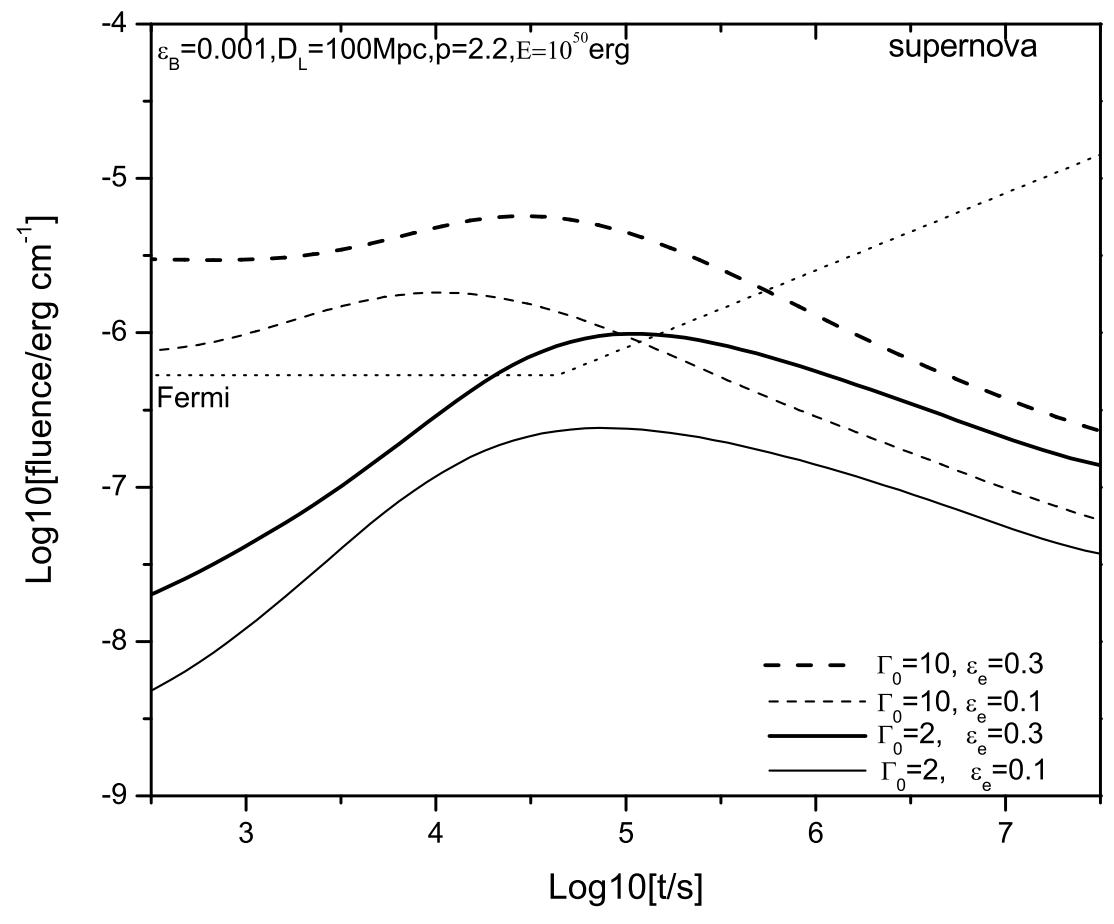

Fig. 6. - Time-integrated fluence (defined as $\int_{0.5 t}^{t} \int_{\nu_{1}}^{\nu_{2}} F_{\nu} d \nu d t$, where $h \nu_{1}=20 \mathrm{MeV}$ and $h \nu_{2}=$ $\left.\min \left(\nu_{\text {cut }}, 300 \mathrm{GeV}\right)\right)$ of the high-energy gamma-ray emission in the two models of low-luminosity GRBs. Short dotted lines represent the fluence threshold of Fermi LAT at $400 \mathrm{MeV}$ with effective detection area $A_{\text {eff }}=6000 \mathrm{~cm}^{2}$. The thicker and thinner solid lines represent the time-integrated fluence in the trans-relativistic ejecta model with $\epsilon_{e}=0.3$ and $\epsilon_{e}=0.1$, respectively; the dashed lines and dotted lines represent the time-integrated fluence in the conventional relativistic ejecta model with $\epsilon_{e}=0.3$ and $\epsilon_{e}=0.1$, respectively. Other parameteres are $E=10^{50} \mathrm{erg}, \dot{m}=1$ and $\epsilon_{B}=0.001$. 


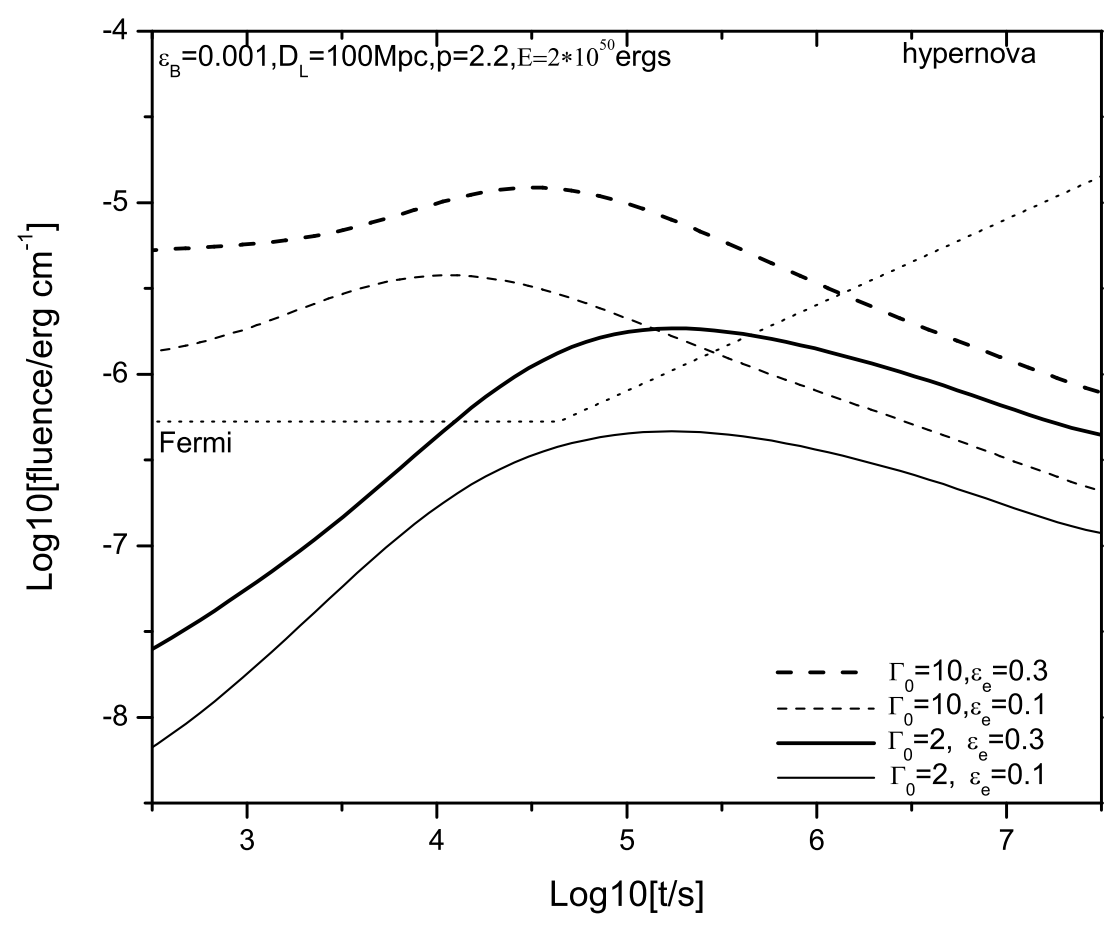

Fig. 7.- Same as Fig, 6, but for the case of a hypernova explosion that has a larger total energy $\left(E=2 \times 10^{50} \mathrm{erg}\right)$, in accordance with the parameters used in Ando \& Mészáros (2008). 